Supporting Information for

\title{
LiOH Formation from Lithium Peroxide Clusters and the Role of lodide Additive
}

Ana E. Torres ${ }^{a, b^{*}}$, Estrella Ramos ${ }^{c}$ and Perla B. Balbuena ${ }^{*}$

aDepartment of Chemical Engineering, Texas A\&M University, College Station, Texas 77843, United States.

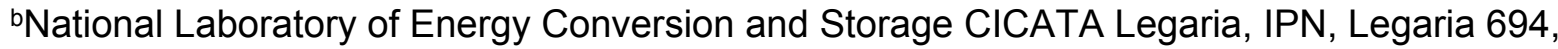
Mexico City, Mexico.

'Materials Research Institute, National Autonomous University of Mexico, Mexico City, Mexico.

*e-mails: aelizabeth.th@gmail.com; balbuena@tamu.edu

\section{S1. Figures and coordinates for first water molecule addition}

$\mathrm{Li}_{2} \mathrm{O}_{2} \_\mathrm{H}_{2} \mathrm{O} \_\mathrm{Li}(3)$

03

$\begin{array}{lccc}\mathrm{O} & 2.71245600 & 3.64434300 & -2.75621800 \\ \mathrm{O} & 2.11834200 & 2.69174800 & -1.75916500 \\ \mathrm{Li} & 4.03732600 & 2.59545700 & -2.09887700 \\ \mathrm{Li} & 0.90537600 & 3.51471500 & -2.88280700 \\ \mathrm{O} & 4.23850100 & 1.01625600 & -3.21788500 \\ \mathrm{O} & 3.62995000 & 1.16615000 & -4.38300000 \\ \mathrm{Li} & 3.12697500 & -0.53456400 & -3.45041100 \\ \mathrm{Li} & 2.40059500 & 2.70978700 & -4.34096000 \\ \mathrm{Li} & 1.29352200 & 1.18523800 & -2.60148600 \\ \mathrm{O} & 1.41572900 & -0.51738200 & -3.27655200 \\ \mathrm{Li} & -0.17494100 & -1.17801100 & -3.30077600 \\ \mathrm{O} & 0.64166300 & 2.23551200 & -4.16386800 \\ \mathrm{Li} & -1.02914100 & 1.77396600 & -4.39976900 \\ \mathrm{Li} & 0.98730300 & 0.51378900 & -4.68748800 \\ \mathrm{O} & -1.11067300 & -0.13739900 & -4.75416600 \\ \mathrm{O} & -1.60775200 & 0.14373100 & -3.55382000 \\ \mathrm{O} & 4.10256400 & 3.17643500 & -0.23080600 \\ \mathrm{H} & 4.21994900 & 2.67631200 & 0.58047100 \\ \mathrm{H} & 3.13863900 & 3.11645900 & -0.49591300\end{array}$

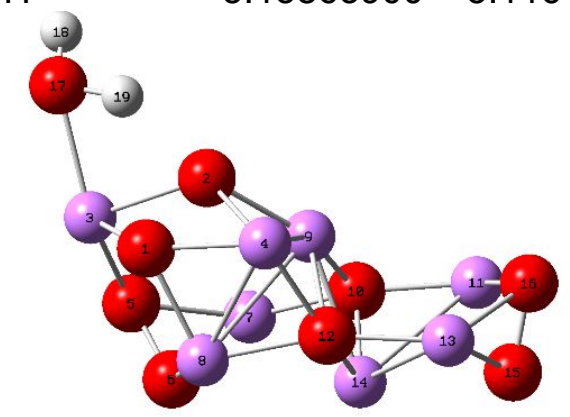




$$
\mathrm{Li}_{2} \mathrm{O}_{2} \mathrm{H}_{2} \mathrm{O} \_\mathrm{Li}(4)
$$

$\begin{array}{cccc}03 & & & \\ \mathrm{O} & 2.90433100 & 3.55134500 & -2.68664200 \\ \mathrm{O} & 2.38861400 & 2.52908000 & -1.71380900 \\ \mathrm{Li} & 4.12368200 & 2.36875200 & -2.13880800 \\ \mathrm{Li} & 1.02583500 & 3.57328800 & -2.81536800 \\ \mathrm{O} & 4.37450500 & 0.85399700 & -3.28525100 \\ \mathrm{O} & 3.73815300 & 1.11679100 & -4.41573700 \\ \mathrm{Li} & 3.13834900 & -0.60645000 & -3.58615800 \\ \mathrm{Li} & 2.50332600 & 2.67476900 & -4.28584300 \\ \mathrm{Li} & 1.37491400 & 1.15396800 & -2.60488000 \\ \mathrm{O} & 1.43803700 & -0.52247800 & -3.36517400 \\ \mathrm{Li} & -0.17707600 & -1.12543500 & -3.30766800 \\ \mathrm{O} & 0.74069300 & 2.28021300 & -4.09125500 \\ \mathrm{Li} & -0.97211000 & 1.91837700 & -4.18762400 \\ \mathrm{Li} & 0.95045300 & 0.57519300 & -4.70534900 \\ \mathrm{O} & -1.15796900 & 0.02425000 & -4.64463300 \\ \mathrm{O} & -1.55395100 & 0.26065800 & -3.39790100 \\ \mathrm{O} & 0.61209700 & 4.19112800 & -0.99071300 \\ \mathrm{H} & 0.89571100 & 5.05923600 & -0.69088500 \\ \mathrm{H} & 1.40448400 & 3.57343100 & -0.92873800\end{array}$

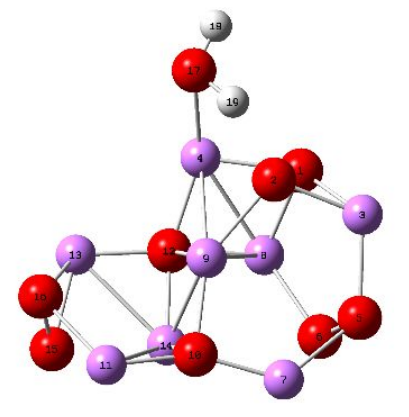

$$
\mathrm{Li}_{2} \mathrm{O}_{2} \mathrm{H}_{2} \mathrm{O} \_\mathrm{Li}(11)
$$

03

$\begin{array}{lccc}\mathrm{O} & 2.86878900 & 3.58893500 & -2.75933400 \\ \mathrm{O} & 2.33745000 & 2.59728000 & -1.75135700 \\ \mathrm{Li} & 4.06688300 & 2.41727700 & -2.16159800 \\ \mathrm{Li} & 1.05932400 & 3.48889900 & -2.74029600 \\ \mathrm{O} & 4.32927600 & 0.88866500 & -3.30396700 \\ \mathrm{O} & 3.66864600 & 1.11019900 & -4.42909700 \\ \mathrm{Li} & 3.10154700 & -0.58328000 & -3.57928200 \\ \mathrm{Li} & 2.41629300 & 2.65226300 & -4.30529100 \\ \mathrm{Li} & 1.36323300 & 1.15320100 & -2.55610100 \\ \mathrm{O} & 1.40734400 & -0.52752700 & -3.28429800 \\ \mathrm{Li} & -0.04747300 & -1.42910500 & -3.11125500 \\ \mathrm{O} & 0.65065400 & 2.25831800 & -4.03040100 \\ \mathrm{Li} & -1.04240500 & 1.87468600 & -4.18758100 \\ \mathrm{Li} & 0.81825600 & 0.52241200 & -4.60708600\end{array}$




$\begin{array}{llrl}\mathrm{O} & -1.30735800 & 0.05565400 & -4.80446200 \\ \mathrm{O} & -1.58162700 & 0.15992300 & -3.50954100 \\ \mathrm{O} & -1.17717400 & -2.58315600 & -4.20939700 \\ \mathrm{H} & -1.98443000 & -3.03096800 & -3.94034300 \\ \mathrm{H} & -1.44327500 & -1.80606400 & -4.74239000\end{array}$

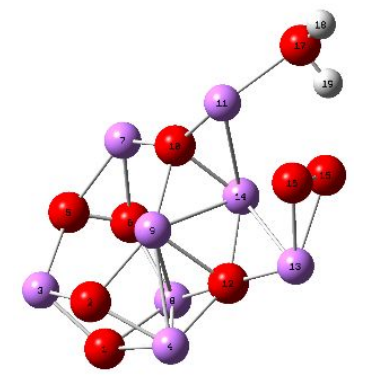

$\mathrm{Li}_{2} \mathrm{O}_{2} \mathrm{H}_{2} \mathrm{O} \_\mathrm{Li}(13)$

$\begin{array}{cccc}03 & & & \\ \mathrm{O} & 2.94310900 & 3.52641000 & -2.62988900 \\ \mathrm{O} & 2.41031900 & 2.47678000 & -1.68294600 \\ \mathrm{Li} & 4.12906400 & 2.29865900 & -2.13409500 \\ \mathrm{Li} & 1.13749500 & 3.46155500 & -2.56698900 \\ \mathrm{O} & 4.33450400 & 0.83642000 & -3.37526000 \\ \mathrm{O} & 3.67367100 & 1.16132300 & -4.47509100 \\ \mathrm{Li} & 3.07242100 & -0.58323300 & -3.73645600 \\ \mathrm{Li} & 2.43865800 & 2.71290400 & -4.22288200 \\ \mathrm{Li} & 1.38892900 & 1.11617000 & -2.57350300 \\ \mathrm{O} & 1.38573700 & -0.50998900 & -3.42575800 \\ \mathrm{Li} & -0.23699600 & -1.08137500 & -3.32984900 \\ \mathrm{O} & 0.66299400 & 2.33208300 & -3.93909400 \\ \mathrm{Li} & -1.07444600 & 2.22478200 & -4.11448900 \\ \mathrm{Li} & 0.79823800 & 0.66090500 & -4.65572900 \\ \mathrm{O} & -1.32438400 & -0.08766200 & -4.64689800 \\ \mathrm{O} & -1.57961900 & 0.33768600 & -3.41486700 \\ \mathrm{O} & -2.55400600 & 2.20089100 & -5.39335400 \\ \mathrm{H} & -3.48703500 & 2.40100200 & -5.27816400 \\ \mathrm{H} & -2.46050400 & 1.23118500 & -5.45857500\end{array}$

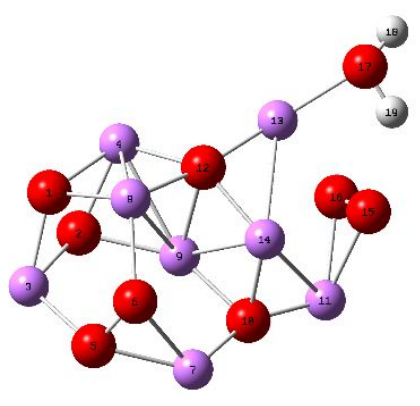


$\mathrm{Li}_{2} \mathrm{O}_{2} \mathrm{H}_{2} \mathrm{O} \_\mathrm{Li}(7)$

$\begin{array}{cccc}03 & & & \\ \mathrm{O} & 3.00845400 & 3.38804100 & -2.78585100 \\ \mathrm{O} & 2.52470000 & 2.25546900 & -1.90853800 \\ \mathrm{Li} & 4.26385700 & 2.22669100 & -2.31299200 \\ \mathrm{Li} & 1.21236300 & 3.31545600 & -2.67043000 \\ \mathrm{O} & 4.74970600 & 0.90641000 & -3.62464000 \\ \mathrm{O} & 3.65805300 & 0.84254100 & -4.36217600 \\ \mathrm{Li} & 2.83292200 & -1.06466400 & -3.96429900 \\ \mathrm{Li} & 2.47420000 & 2.45234700 & -4.28884300 \\ \mathrm{Li} & 1.42314300 & 0.92859400 & -2.77228500 \\ \mathrm{O} & 1.17912000 & -0.66506100 & -3.66506500 \\ \mathrm{Li} & -0.47026900 & -1.12333600 & -3.46227500 \\ \mathrm{O} & 0.66046000 & 2.22921200 & -4.04659900 \\ \mathrm{Li} & -1.07126700 & 2.01972900 & -4.05340100 \\ \mathrm{Li} & 0.64409600 & 0.58200100 & -4.82747500 \\ \mathrm{O} & -1.48018600 & 0.19440300 & -4.62159300 \\ \mathrm{O} & -1.75170100 & 0.36554000 & -3.33169400 \\ \mathrm{O} & 4.63949300 & -1.71675100 & -3.75276700 \\ \mathrm{H} & 5.23611700 & -2.32597600 & -4.19267800 \\ \mathrm{H} & 5.05243100 & -0.82233600 & -3.72047300\end{array}$

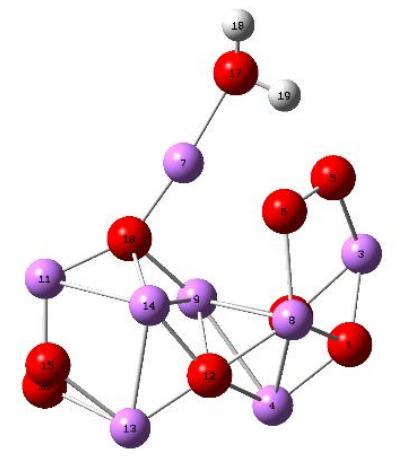

$\mathrm{Li}_{2} \mathrm{O}_{2} \mathrm{H}_{2} \mathrm{O} \_\mathrm{Li}(8)$

03

$\begin{array}{llll}\mathrm{O} & 2.71253400 & 3.72310500 & -2.77843400 \\ \mathrm{O} & 2.39878200 & 2.63292400 & -1.78255800 \\ \mathrm{Li} & 4.06883300 & 2.62544100 & -2.39721700 \\ \mathrm{Li} & 0.91207100 & 3.50519500 & -2.33267600 \\ \mathrm{O} & 4.63715200 & 1.03096700 & -3.29332500 \\ \mathrm{O} & 4.57562400 & 0.94022400 & -4.60842100 \\ \mathrm{Li} & 3.37883200 & -0.36912900 & -3.82601100 \\ \mathrm{Li} & 1.82080300 & 3.38130400 & -4.36014200 \\ \mathrm{Li} & 1.44607800 & 1.29407400 & -2.75819500 \\ \mathrm{O} & 1.68006300 & -0.14596900 & -3.84976800 \\ \mathrm{Li} & 0.19387000 & -1.01355000 & -3.91119800 \\ \mathrm{O} & 0.34501900 & 2.53676500 & -3.78507300\end{array}$




$\begin{array}{lccc}\mathrm{Li} & -1.22152500 & 1.81805000 & -3.94834500 \\ \mathrm{Li} & 1.00957300 & 1.15371300 & -4.91617200 \\ \mathrm{O} & -1.11181200 & 0.11682400 & -4.89849900 \\ \mathrm{O} & -1.47229400 & -0.05999200 & -3.63047800 \\ \mathrm{O} & 2.60370600 & 2.29265200 & -5.77314400 \\ \mathrm{H} & 3.42884000 & 1.88905600 & -5.39729800 \\ \mathrm{H} & 2.78527900 & 2.50498300 & -6.69415100\end{array}$

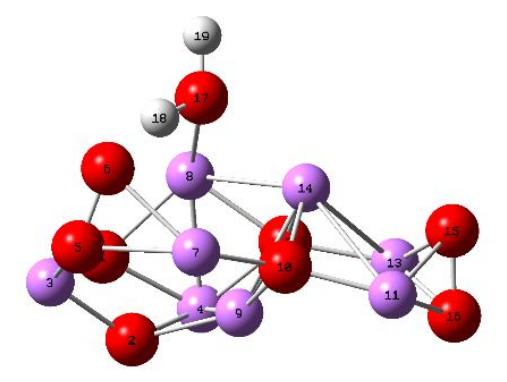

$\mathrm{Li}_{2} \mathrm{O}_{2} \_\mathrm{H}_{2} \mathrm{O} \_\mathrm{Li}(9)$

03

$\begin{array}{cccc}\mathrm{O} & 2.63274400 & 3.44957200 & -2.42556100 \\ \mathrm{O} & 2.03280800 & 2.30171300 & -1.66069800 \\ \mathrm{Li} & 3.80719600 & 2.22637700 & -1.86430500 \\ \mathrm{Li} & 0.85139900 & 3.26479200 & -2.70845800 \\ \mathrm{O} & 4.30602400 & 0.88207800 & -3.13822900 \\ \mathrm{O} & 3.80926600 & 1.26060600 & -4.30505300 \\ \mathrm{Li} & 3.21574100 & -0.57130500 & -3.85537100 \\ \mathrm{Li} & 2.42563700 & 2.66325100 & -4.11824700 \\ \mathrm{Li} & 1.19055100 & 0.84736900 & -2.73236800 \\ \mathrm{O} & 1.50569200 & -0.63408100 & -3.82063200 \\ \mathrm{Li} & -0.08118600 & -1.28794100 & -3.68135500 \\ \mathrm{O} & 0.64213200 & 2.21383200 & -4.16912600 \\ \mathrm{Li} & -1.03498800 & 1.75320400 & -4.34361200 \\ \mathrm{Li} & 0.99903500 & 0.61503000 & -4.97209100 \\ \mathrm{O} & -1.22482600 & -0.09565200 & -4.82456300 \\ \mathrm{O} & -1.53990400 & 0.07202600 & -3.54404600 \\ \mathrm{O} & 0.10177400 & 0.60216100 & -1.04336600 \\ \mathrm{H} & 0.54939000 & 1.38922200 & -0.68651300 \\ \mathrm{H} & -0.84742000 & 0.75939900 & -1.02384200\end{array}$

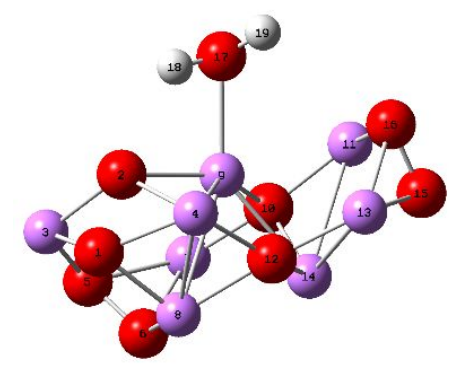




$\begin{array}{lccc}\mathrm{Li}_{2} \mathrm{O}_{2} \mathrm{H}_{2} \mathrm{O}_{-} \mathrm{Li}(14) & & \\ \mathrm{O} 3 & & & \\ \mathrm{O} & 2.78961100 & 3.13040300 & -4.01060000 \\ \mathrm{O} & 2.40659200 & 2.61169100 & -2.64799200 \\ \mathrm{Li} & 4.06553000 & 2.15505900 & -3.16799600 \\ \mathrm{Li} & 0.99922500 & 3.31966800 & -3.53828900 \\ \mathrm{O} & 4.49510400 & 0.28545800 & -2.69018900 \\ \mathrm{O} & 4.36451000 & 0.32353900 & -4.01107600 \\ \mathrm{Li} & 3.10032600 & -0.92860500 & -3.04594200 \\ \mathrm{Li} & 1.81761100 & 2.20292600 & -5.27635700 \\ \mathrm{Li} & 1.31071700 & 1.09575700 & -2.96875000 \\ \mathrm{O} & 1.43402000 & -0.68312100 & -3.37854900 \\ \mathrm{Li} & -0.10171100 & -1.37712000 & -2.99575200 \\ \mathrm{O} & 0.26646400 & 1.87560400 & -4.41536200 \\ \mathrm{Li} & -1.36220600 & 1.32882300 & -4.18810100 \\ \mathrm{Li} & 0.68159000 & 0.08107400 & -4.84831800 \\ \mathrm{O} & -1.39803700 & -0.63699600 & -4.32018500 \\ \mathrm{O} & -1.66048000 & -0.22475700 & -3.08374300 \\ \mathrm{O} & 2.37773900 & 0.44421800 & -5.98029300 \\ \mathrm{H} & 2.96761300 & 0.12339300 & -5.26543500 \\ \mathrm{H} & 2.77977200 & 0.19745700 & -6.81895500\end{array}$

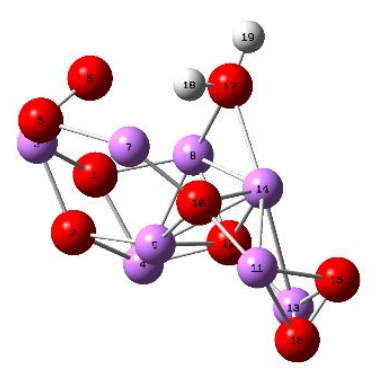

\section{$\mathrm{LI}_{2} \mathrm{O}_{2}$ tetramer triplet}

$\begin{array}{cccc}03 & & & \\ \mathrm{O} & 2.27918800 & -1.56660100 & 0.09925000 \\ \mathrm{O} & 1.57629600 & -1.17016500 & -1.17772200 \\ \mathrm{Li} & 2.97183200 & -0.15525700 & -0.72939900 \\ \mathrm{Li} & 0.62806900 & -2.28906800 & -0.07389600 \\ \mathrm{O} & 2.34907800 & 1.53732800 & -0.04802800 \\ \mathrm{O} & 1.64061900 & 1.31207000 & 1.04682300 \\ \mathrm{Li} & 0.61871800 & 2.35109500 & -0.31002300 \\ \mathrm{Li} & 1.22283800 & -0.61914900 & 1.29505600 \\ \mathrm{Li} & -0.02772500 & -0.16347700 & -0.86794300 \\ \mathrm{O} & -0.82629000 & 1.48252800 & -0.64758400 \\ \mathrm{Li} & -2.49946600 & 1.28405100 & -0.99977900 \\ \mathrm{O} & -0.47768600 & -1.10267400 & 0.80527700 \\ \mathrm{Li} & -2.17560500 & -1.48740800 & 0.69426600 \\ \mathrm{Li} & -1.07786400 & 0.62312300 & 0.91499300 \\ \mathrm{O} & -3.19322800 & 0.17839200 & 0.54679300 \\ \mathrm{O} & -3.22077800 & -0.49984500 & -0.59603800\end{array}$




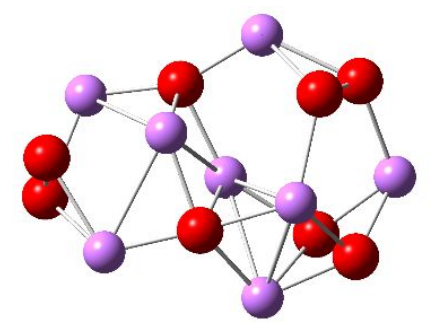

$\mathrm{LI}_{6} \mathrm{O}_{6}$.LiOH.LiOOH

03

$\begin{array}{lccc}\mathrm{O} & -2.32053600 & 0.73076700 & 0.78516700 \\ \mathrm{O} & -2.13143800 & 0.68561300 & -0.68224200 \\ \mathrm{Li} & -3.21422400 & -0.66988100 & 0.03843200 \\ \mathrm{Li} & -0.65733100 & 1.84799200 & 1.10051700 \\ \mathrm{O} & -1.88514900 & -2.03353700 & -0.33846000 \\ \mathrm{O} & -0.99800700 & -1.99452700 & 0.63599300 \\ \mathrm{Li} & -0.88352500 & -0.75392800 & -1.55074100 \\ \mathrm{Li} & -0.98801900 & -0.34517100 & 1.64217200 \\ \mathrm{Li} & 0.73541700 & 1.03665500 & -0.59121600 \\ \mathrm{O} & 0.84466700 & -0.53328300 & -1.51043300 \\ \mathrm{Li} & 2.57683200 & -0.64475400 & -1.55767000 \\ \mathrm{O} & 0.55197000 & 0.47643200 & 1.28848000 \\ \mathrm{Li} & 2.27247500 & 0.50900900 & 1.59936300 \\ \mathrm{Li} & 0.83399200 & -1.07589400 & 0.22059200 \\ \mathrm{O} & 3.12183900 & -0.81186900 & 0.38224300 \\ \mathrm{O} & 3.33610800 & 0.42368700 & -0.05662100 \\ \mathrm{O} & -0.49923900 & 2.46480700 & -0.63938400 \\ \mathrm{H} & -0.57286600 & 3.29031400 & -1.12189100 \\ \mathrm{H} & -1.50704400 & 1.55845400 & -0.82155400 \\ & & & \end{array}$

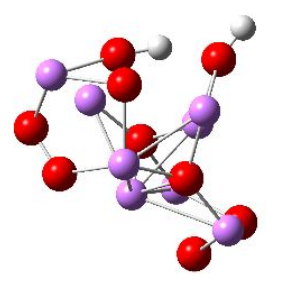

$\mathrm{Li}_{8} \mathrm{O}_{6} \mathrm{H}_{2} \mathrm{O}$

01

\begin{tabular}{|c|c|c|c|}
\hline & -2.27179700 & 0.07082500 & -0.81503600 \\
\hline & -2.50271300 & 0.44385300 & 0.61485200 \\
\hline & -3.98306200 & 0.25181500 & -0.38377500 \\
\hline & -0.98796700 & -1.51222200 & -0.87604600 \\
\hline & -1.17884500 & 1.77707500 & 0.81952000 \\
\hline & -0.56269200 & 0.69165000 & -1.25054200 \\
\hline & 0.54093300 & -0.33212600 & 0.53415800 \\
\hline & 0.39651200 & 1.55859800 & 0.11699600 \\
\hline & & & \\
\hline
\end{tabular}




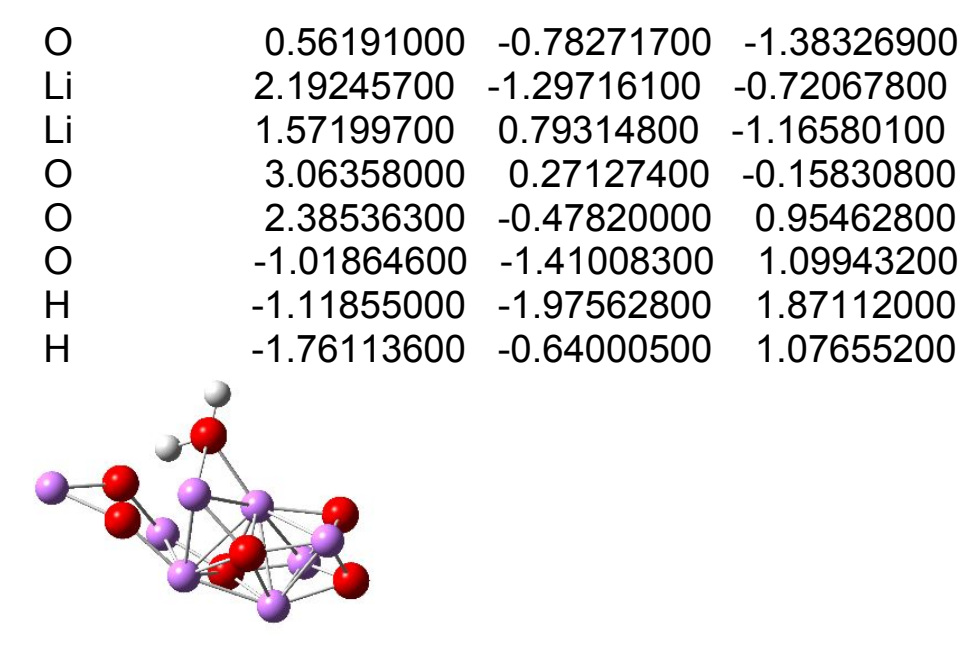

S2. Figures and coordinates for Second water addition

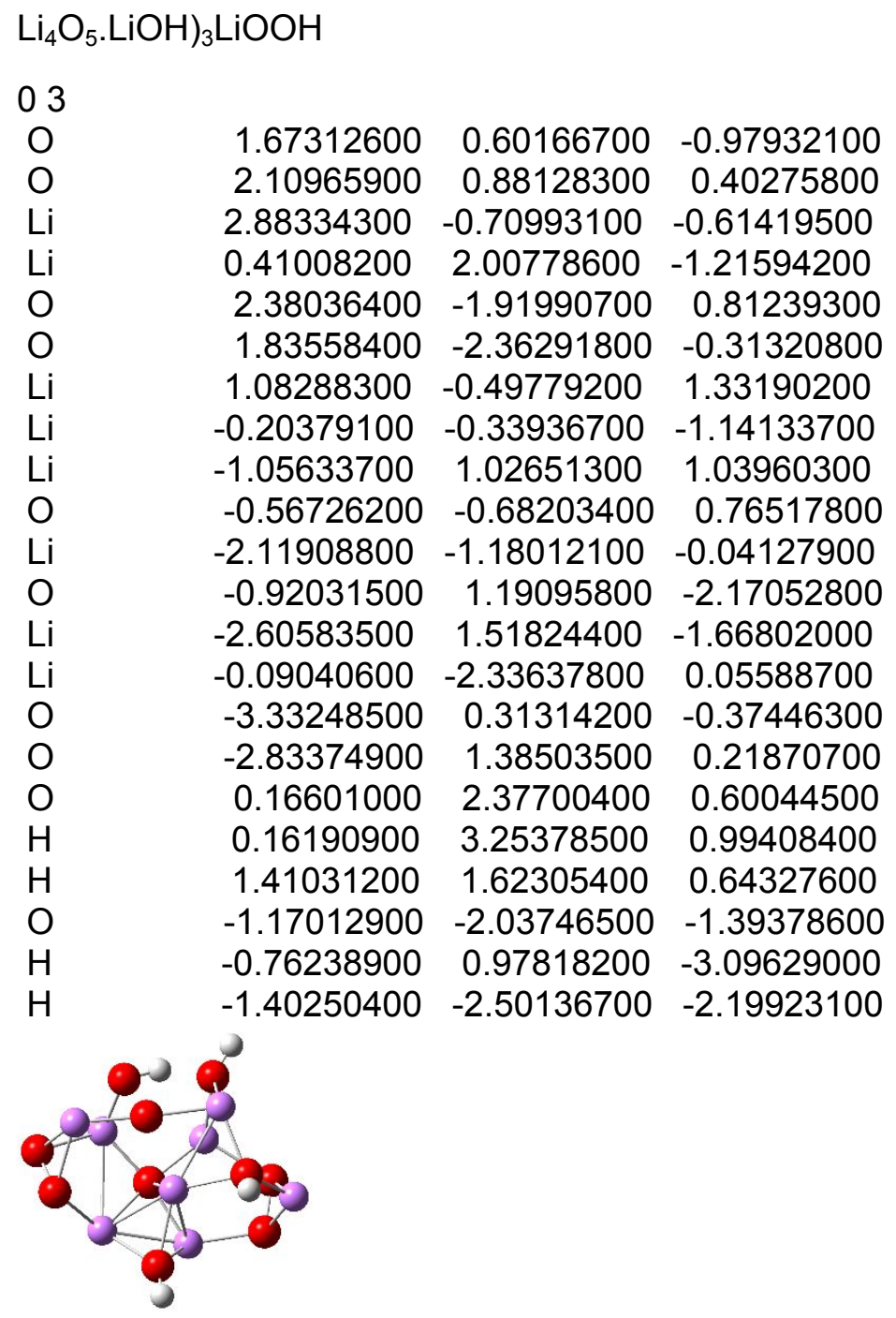




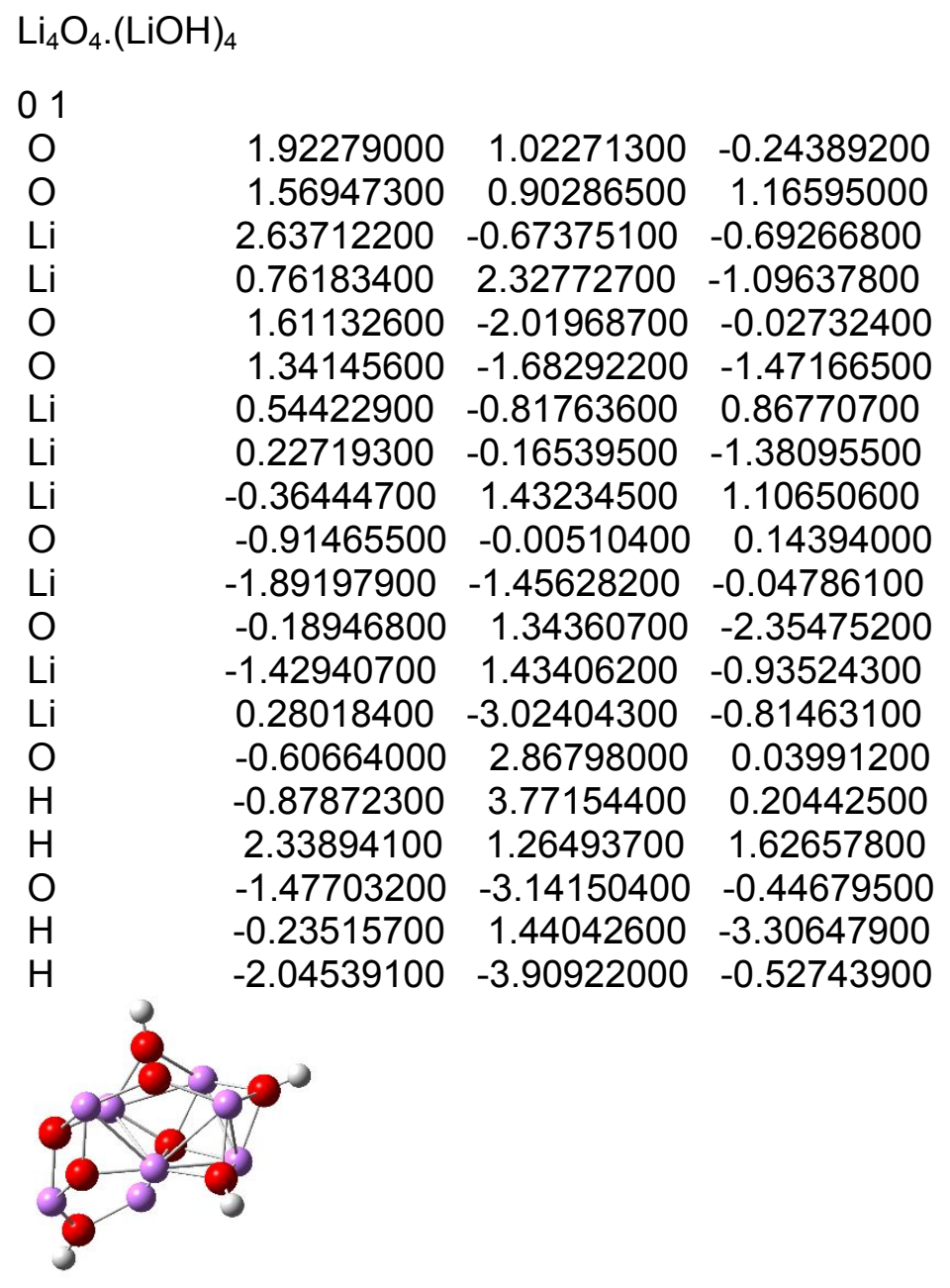

S3. Figures and coordinates for Third water addition

$\mathrm{Li}_{2} \mathrm{O}_{4} \cdot(\mathrm{LiOH})_{5} \cdot \mathrm{LiOOH}$

$\begin{array}{lccc}03 & & & \\ \mathrm{O} & -0.86907500 & -0.96655400 & 1.67951400 \\ \mathrm{O} & -1.11710600 & 0.47605800 & 1.56874400 \\ \mathrm{Li} & -2.65254400 & -0.71526200 & 1.26889100 \\ \mathrm{Li} & 0.91877500 & -0.84579800 & 2.35967200 \\ \mathrm{O} & -3.22063000 & 0.31854500 & -0.29265600 \\ \mathrm{O} & -2.89136000 & -0.92115800 & -0.62331300 \\ \mathrm{Li} & -1.51752300 & 1.45258900 & -0.18828900 \\ \mathrm{Li} & 0.14515800 & -1.92683700 & 0.31488800 \\ \mathrm{Li} & 0.88200300 & 1.73508800 & -0.25564700 \\ \mathrm{O} & -0.34234100 & 0.67948000 & -1.52407000 \\ \mathrm{Li} & 1.03568200 & -0.52003200 & -1.86538600 \\ \mathrm{O} & 1.80409300 & -1.93630700 & 1.18363800 \\ \mathrm{Li} & 2.53825500 & -0.37128300 & 0.57064600 \\ \mathrm{Li} & -1.37971100 & -0.84987900 & -1.79217300 \\ \mathrm{O} & 2.71977800 & 0.17747700 & -1.26519300\end{array}$




$\begin{array}{lrrr}\mathrm{O} & 2.60881200 & 1.49681300 & -1.24209600 \\ \mathrm{O} & 1.38527000 & 0.79843300 & 1.47253800 \\ \mathrm{H} & 1.72876400 & 1.56135800 & 1.95163500 \\ \mathrm{H} & -0.16293800 & 0.83272700 & 1.54927400 \\ \mathrm{O} & 0.00435800 & -2.01588500 & -1.52607000 \\ \mathrm{H} & 2.28407800 & -2.76656000 & 1.21564400 \\ \mathrm{H} & 0.15125600 & -2.89777000 & -1.87726500 \\ \mathrm{O} & -0.47783600 & 2.90420700 & 0.06580800 \\ \mathrm{H} & -0.55231900 & 3.70193300 & 0.59037300 \\ \mathrm{H} & -0.38998100 & 1.43778500 & -2.12131300\end{array}$

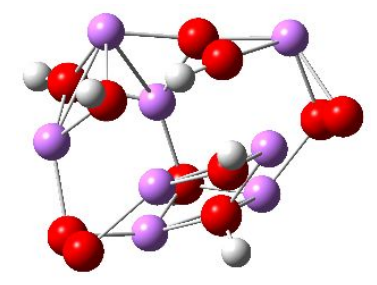

$\mathrm{Li}_{4} \mathrm{O}_{2} \cdot(\mathrm{LiOH})_{4} \cdot \mathrm{H}_{2} \mathrm{O}$

01

O $\quad 0.72203500 \quad-1.56511900 \quad-1.00976000$

$\begin{array}{llll}\mathrm{O} & -0.17954300 & -0.67708200 & -1.81108000\end{array}$

Li $\quad \begin{array}{llll}0.47345400 & -2.08310800 & -2.67681000\end{array}$

Li $\quad 0.06873200 \quad-1.61889300 \quad 0.81027700$

$\begin{array}{lllll}\mathrm{Li} & -1.22882400 & 0.56180400 & -0.72501300\end{array}$

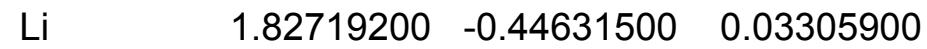

Li $\quad-1.65566500 \quad 1.00326400 \quad 1.59664800$

$\begin{array}{llll}\mathrm{O} & -0.45392100 & 2.15799200 & -1.33431000\end{array}$

Li $\quad \begin{array}{llll}0.71299000 & 2.01722400 & 0.14064300\end{array}$

$\begin{array}{llll}\mathrm{O} & 1.75965100 & -1.41817200 & 1.70427400\end{array}$

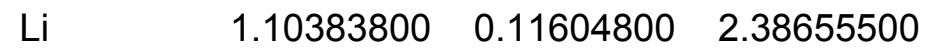

Li $\quad 0.80880900 \quad 0.94597400 \quad-1.95382900$

$\begin{array}{llll}\mathrm{O} & -0.03712400 & 0.39473800 & 0.90096600\end{array}$

$\begin{array}{llll}\mathrm{O} & 0.08415300 & 1.53997800 & 1.86791800\end{array}$

$\begin{array}{llll}\mathrm{O} & -1.77957500 & -2.04465100 & 0.30236100\end{array}$

$\begin{array}{llll}\mathrm{H} & -2.31243000 & -1.18333100 & 0.38322500\end{array}$

$\mathrm{H} \quad-1.45377000-2.00852300 \quad-0.61212300$

$\begin{array}{llll}\mathrm{O} & 2.20234000 & 1.18550300 & -0.72307100\end{array}$

$\begin{array}{llll}\mathrm{H} & 2.37857700 & -2.08247100 & 2.01137000\end{array}$

$\begin{array}{llll}\mathrm{H} & 3.05761300 & 1.57560100 & -0.91157700\end{array}$

$\begin{array}{llll}\mathrm{O} & -2.79072700 & 0.32057000 & 0.36448300\end{array}$

$\begin{array}{llll}\mathrm{H} & -3.70992400 & 0.48428900 & 0.14337300\end{array}$

$\begin{array}{llll}\mathrm{H} & -0.91165400 & 2.92008200 & -1.68961100\end{array}$ 


\section{S4. Figures and coordinates for Fourth water addition}

$\begin{array}{cccc}\mathrm{Li}_{2} \mathrm{O}_{4} \cdot(\mathrm{LiOH})_{5} \mathrm{LiOOH} . \mathrm{H}_{2} \mathrm{O} & & \\ 03 & & & \\ \mathrm{O} & -0.77431600 & -1.10701200 & 1.62463000 \\ \mathrm{O} & -1.08626400 & 0.32329000 & 1.56252300 \\ \mathrm{Li} & -2.62465600 & -0.96441400 & 1.18960900 \\ \mathrm{Li} & 1.02677200 & -0.95013300 & 2.26036400 \\ \mathrm{O} & -3.18057200 & 0.17866900 & -0.31450300 \\ \mathrm{O} & -2.82014400 & -1.02685800 & -0.72742900 \\ \mathrm{Li} & -1.51167800 & 1.34167100 & -0.15359200 \\ \mathrm{Li} & 0.24364500 & -1.97237200 & 0.19429200 \\ \mathrm{Li} & 0.87420900 & 1.73241600 & -0.20075900 \\ \mathrm{O} & -0.31334800 & 0.69279200 & -1.52391200 \\ \mathrm{Li} & 1.09209000 & -0.46199500 & -1.91628500 \\ \mathrm{O} & 1.92430900 & -1.95446600 & 1.02591600 \\ \mathrm{Li} & 2.60585600 & -0.33215800 & 0.50033500 \\ \mathrm{Li} & -1.31723200 & -0.84487000 & -1.87882800 \\ \mathrm{O} & 2.75625000 & 0.29057800 & -1.31537300 \\ \mathrm{O} & 2.59910900 & 1.60328300 & -1.23790300 \\ \mathrm{O} & 1.42990400 & 0.74635500 & 1.46776200 \\ \mathrm{H} & 1.74854700 & 1.49941500 & 1.97838700 \\ \mathrm{H} & -0.15709200 & 0.72349000 & 1.55859900 \\ \mathrm{O} & 0.09548900 & -1.98917500 & -1.65038200 \\ \mathrm{H} & 2.43415800 & -2.76694400 & 0.99852700 \\ \mathrm{H} & 0.26391200 & -2.85203400 & -2.03736200 \\ \mathrm{O} & -0.53688500 & 2.82322600 & 0.18745000 \\ \mathrm{H} & -0.64768700 & 3.58324300 & 0.75939700 \\ \mathrm{H} & -0.38186300 & 1.47775700 & -2.08340000 \\ \mathrm{O} & -3.18287700 & -1.22731500 & 3.01583000 \\ \mathrm{H} & -3.72669500 & -0.60979300 & 3.52133900 \\ \mathrm{H} & -2.26517000 & -1.10896900 & 3.31032800 \\ & & & \\ & & & \\ & & & \\ & & & \\ & & & \\ & & & \end{array}$

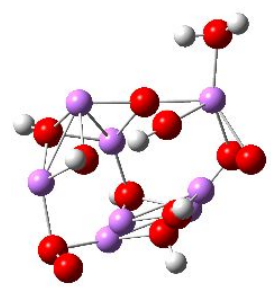

$\mathrm{Li}_{2} \mathrm{O}_{3} \cdot(\mathrm{LiOH})_{6} \cdot \mathrm{H}_{2} \mathrm{O}$

01

\begin{tabular}{|c|c|c|c|}
\hline & -0.66546400 & -0.05546400 & 1.48527800 \\
\hline 0 & -1.70173600 & 0.14585400 & 2.49004000 \\
\hline & -2.35540900 & -0.95477800 & 1.08364200 \\
\hline & 1.14415900 & -0.20724400 & 1.78043100 \\
\hline & -2.88813500 & -0.41206300 & -0.55740100 \\
\hline & -2.01932600 & -1.63074300 & \\
\hline
\end{tabular}




$\begin{array}{cccc}\mathrm{Li} & -2.44589600 & 1.35793000 & -0.18573300 \\ \mathrm{Li} & -0.18699200 & -1.50271900 & 0.04544900 \\ \mathrm{Li} & -0.28560300 & 1.67279900 & 0.64802900 \\ \mathrm{O} & -0.87919000 & 1.62923100 & -1.27402800 \\ \mathrm{Li} & 0.84388600 & 1.03983500 & -1.43914500 \\ \mathrm{O} & 1.53730000 & -1.83318000 & 0.99258600 \\ \mathrm{Li} & 2.11466800 & -0.74255200 & -0.43682800 \\ \mathrm{Li} & -1.34428600 & -0.24595700 & -1.63820900 \\ \mathrm{O} & 1.59742100 & 1.00592000 & 0.31471400 \\ \mathrm{H} & 2.33886100 & 1.59325500 & 0.49751800 \\ \mathrm{H} & -1.94866900 & 1.11245500 & 2.22500500 \\ \mathrm{O} & 0.63535500 & -0.76967700 & -1.60994300 \\ \mathrm{H} & 1.46554500 & -2.67002500 & 1.45891000 \\ \mathrm{H} & 0.70024000 & -1.41104800 & -2.32416600 \\ \mathrm{O} & -1.96359100 & 2.31865200 & 1.32843400 \\ \mathrm{H} & -2.29942200 & 3.14434400 & 1.68195300 \\ \mathrm{H} & -1.07258000 & 2.46517500 & -1.71074700 \\ \mathrm{O} & 3.96383900 & -1.30793100 & -0.04618400 \\ \mathrm{H} & 3.42295600 & -1.83034200 & 0.58189100 \\ \mathrm{H} & 4.54878300 & -1.91830500 & -0.51024300\end{array}$

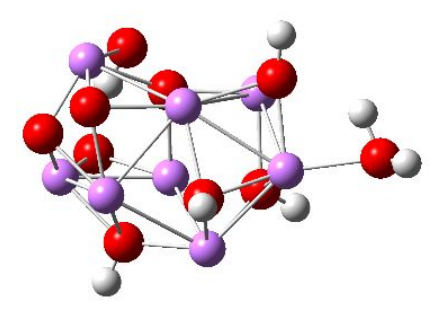

$\mathrm{Li}_{2} \mathrm{O}_{2} \mathrm{H}_{2} \mathrm{O}_{-} \mathrm{Li}(4)$ other site

03

$\begin{array}{lccc}\mathrm{O} & 3.11030300 & 3.59163100 & -2.75001400 \\ \mathrm{O} & 2.18305000 & 2.55464500 & -2.17821100 \\ \mathrm{Li} & 3.91428000 & 2.26824900 & -1.82015700 \\ \mathrm{Li} & 1.34308600 & 4.13951400 & -2.72096500 \\ \mathrm{O} & 4.32766000 & 0.59950500 & -2.69893700 \\ \mathrm{O} & 4.10428500 & 0.91424400 & -3.96550300 \\ \mathrm{Li} & 3.05344200 & -0.66075300 & -3.53034300 \\ \mathrm{Li} & 2.92515400 & 2.49572700 & -4.16591700 \\ \mathrm{Li} & 1.20879200 & 1.25509900 & -3.07269600 \\ \mathrm{O} & 1.36992200 & -0.43154400 & -3.83148000 \\ \mathrm{Li} & -0.30097600 & -0.65533500 & -3.41361100 \\ \mathrm{O} & 1.23025400 & 2.24388600 & -4.95112400 \\ \mathrm{Li} & -0.58882200 & 2.71538000 & -4.13063700 \\ \mathrm{Li} & 1.17247100 & 0.49473100 & -5.31873700 \\ \mathrm{O} & -1.32552800 & 0.83424500 & -4.18561000 \\ \mathrm{O} & -0.87037500 & 1.14863800 & -2.97874300 \\ \mathrm{O} & -0.12577300 & 4.39376400 & -3.73565700 \\ \mathrm{H} & 1.08682700 & 3.12977900 & -5.30763100 \\ \mathrm{H} & -0.68184400 & 5.17382000 & -3.71711100\end{array}$




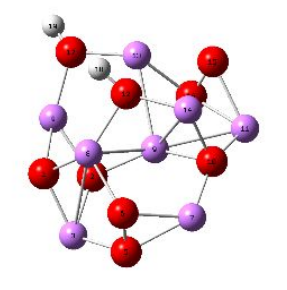

$\mathrm{Li}_{2} \mathrm{O}_{2} \mathrm{H}_{2} \mathrm{O} \_\mathrm{Li}(3)$ other site

$\begin{array}{cccc}03 & & & \\ \mathrm{O} & 2.85623300 & 3.44156500 & -2.63459200 \\ \mathrm{O} & 2.31895500 & 2.39037900 & -1.69324300 \\ \mathrm{Li} & 4.05343600 & 2.24656700 & -2.08384000 \\ \mathrm{Li} & 1.04903300 & 3.32366000 & -2.63605400 \\ \mathrm{O} & 4.34745600 & 0.84655700 & -3.39231300 \\ \mathrm{O} & 3.55503800 & 1.04196800 & -4.43325900 \\ \mathrm{Li} & 3.90478000 & -0.85639800 & -4.16771600 \\ \mathrm{Li} & 2.42994900 & 2.62912000 & -4.24780200 \\ \mathrm{Li} & 1.38252200 & 0.99214500 & -2.57479300 \\ \mathrm{O} & 1.00007900 & -0.66302000 & -3.29666600 \\ \mathrm{Li} & -0.71150200 & -1.07646700 & -3.40513500 \\ \mathrm{O} & 0.68758600 & 2.16324800 & -4.02380000 \\ \mathrm{Li} & -1.01855800 & 1.99367300 & -4.32102500 \\ \mathrm{Li} & 0.88712200 & 0.48773000 & -4.71438700 \\ \mathrm{O} & -1.38149000 & 0.13909000 & -4.79262900 \\ \mathrm{O} & -1.87535600 & 0.43698500 & -3.59458700 \\ \mathrm{O} & 2.92704100 & -2.24363300 & -4.17456700 \\ \mathrm{H} & 1.66224500 & -1.38507800 & -3.48037800 \\ \mathrm{H} & 2.94929000 & -3.19904300 & -4.19980100\end{array}$

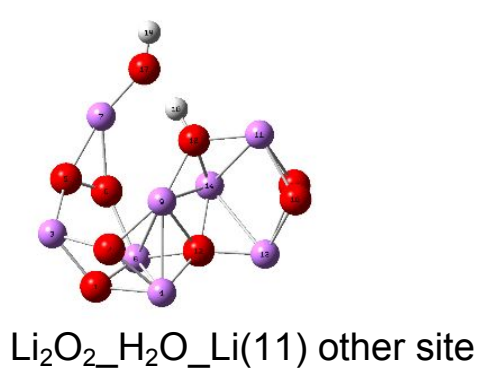

03

$\begin{array}{lccc}\mathrm{O} & 2.76160200 & 3.37759400 & -2.45139500 \\ \mathrm{O} & 2.47778400 & 2.14038300 & -1.63348200 \\ \mathrm{Li} & 4.17897900 & 2.36036400 & -2.11177400 \\ \mathrm{Li} & 0.99848400 & 2.99063700 & -2.31838900 \\ \mathrm{O} & 4.57351800 & 1.12414800 & -3.55865900 \\ \mathrm{O} & 3.79858600 & 1.43468000 & -4.58491900 \\ \mathrm{Li} & 3.63393900 & -0.43236700 & -4.18098500 \\ \mathrm{Li} & 2.32524600 & 2.67040600 & -4.11226300 \\ \mathrm{Li} & 1.63699400 & 0.75724900 & -2.62873000 \\ \mathrm{O} & 1.96344500 & -0.69331800 & -3.75317600\end{array}$




$\begin{array}{cccc}\mathrm{Li} & -1.17538500 & -1.80232400 & -3.97280000 \\ \mathrm{O} & 0.66091300 & 1.96223700 & -3.81290800 \\ \mathrm{Li} & -1.01872000 & 1.69679700 & -4.16617700 \\ \mathrm{Li} & 0.86066800 & 0.37643700 & -4.71523300 \\ \mathrm{O} & -1.10747800 & -0.13102600 & -4.97990300 \\ \mathrm{O} & -1.89008800 & 0.02964900 & -3.91879000 \\ \mathrm{O} & 0.19406100 & -2.64161300 & -3.42366500 \\ \mathrm{H} & 1.43694500 & -1.52484600 & -3.58440700 \\ \mathrm{H} & 0.42851100 & -3.47467500 & -3.01593200\end{array}$

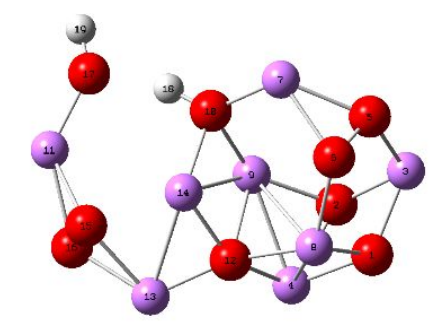

$\mathrm{Li}_{2} \mathrm{O}_{2} \mathrm{H}_{2} \mathrm{O} \_\mathrm{Li}(13)$ other site

$\begin{array}{cccc}03 & & & \\ \mathrm{O} & 2.06875800 & 3.65807800 & -2.61990900 \\ \mathrm{O} & 1.47842500 & 2.33704600 & -2.23979100 \\ \mathrm{Li} & 3.30032500 & 2.50350500 & -2.00082400 \\ \mathrm{Li} & 0.25393600 & 3.76195400 & -2.32377800 \\ \mathrm{O} & 4.12751800 & 0.92955100 & -2.65549700 \\ \mathrm{O} & 3.79031100 & 1.44221200 & -3.83303300 \\ \mathrm{Li} & 3.29090600 & -0.45460100 & -3.71250100 \\ \mathrm{Li} & 1.96865900 & 2.61554600 & -4.11685400 \\ \mathrm{Li} & 1.07371400 & 0.63011600 & -2.66402900 \\ \mathrm{O} & 1.58548200 & -0.68789200 & -3.79589900 \\ \mathrm{Li} & 0.00034500 & -1.36972900 & -3.53825900 \\ \mathrm{O} & 0.66311200 & 1.99688800 & -5.25286900 \\ \mathrm{Li} & -0.92706000 & 2.12099400 & -4.12977000 \\ \mathrm{Li} & 0.94882300 & 0.22049100 & -5.19398100 \\ \mathrm{O} & -1.21091200 & -0.16689200 & -4.38256200 \\ \mathrm{O} & -0.94920300 & 0.34433500 & -3.18788500 \\ \mathrm{H} & 0.50477600 & 2.54471500 & -6.02636100 \\ \mathrm{O} & -1.24378400 & 3.63224800 & -3.24624500 \\ \mathrm{H} & -2.05448500 & 4.13809200 & -3.19831100\end{array}$

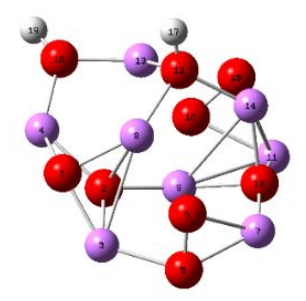


$\mathrm{Li}_{2} \mathrm{O}_{2} \mathrm{H}_{2} \mathrm{O} \_\mathrm{Li}(8)$ other site

$\begin{array}{cccc}03 & & & \\ \mathrm{O} & 3.11409000 & 3.38525500 & -2.70338100 \\ \mathrm{O} & 2.28397100 & 2.39178300 & -1.93018700 \\ \mathrm{Li} & 4.03248100 & 2.17509200 & -1.73685800 \\ \mathrm{Li} & 1.35548300 & 3.78869600 & -2.65677800 \\ \mathrm{O} & 4.55576300 & 0.61883800 & -2.70350500 \\ \mathrm{O} & 4.49051300 & 1.01577000 & -3.96441200 \\ \mathrm{Li} & 3.17883500 & -0.35851800 & -3.65014500 \\ \mathrm{Li} & 3.45628000 & 2.68873400 & -4.38180700 \\ \mathrm{Li} & 1.20227700 & 1.45261600 & -3.17030900 \\ \mathrm{O} & 1.55328200 & -0.04415100 & -4.16711500 \\ \mathrm{Li} & -0.03442500 & -0.71786600 & -4.09781200 \\ \mathrm{O} & 0.24971400 & 3.02472800 & -3.90879900 \\ \mathrm{Li} & -1.30274400 & 2.19775200 & -4.09442400 \\ \mathrm{Li} & 1.76980600 & 0.90959800 & -5.62938500 \\ \mathrm{O} & -1.49559400 & 0.41311800 & -4.71009200 \\ \mathrm{O} & -1.40335300 & 0.44388200 & -3.38301800 \\ \mathrm{O} & 2.38232100 & 2.56472000 & -5.83995800 \\ \mathrm{H} & 2.52270900 & 3.06788100 & -6.64368900 \\ \mathrm{H} & 0.77566500 & 3.12290600 & -4.72362700\end{array}$

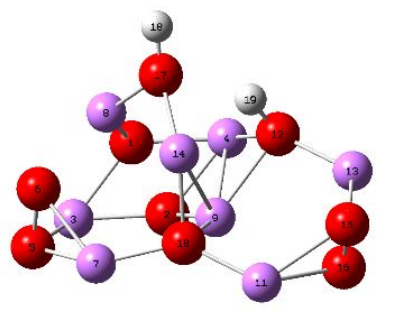

$\mathrm{Li}_{2} \mathrm{O}_{2} \mathrm{H}_{2} \mathrm{O} \_\mathrm{Li}(9)$ other site

03

$\begin{array}{lccc}\mathrm{O} & 2.96858200 & 3.82482100 & -2.96541400 \\ \mathrm{O} & 2.61476400 & 3.02605200 & -1.73591300 \\ \mathrm{Li} & 4.22843700 & 2.69914000 & -2.39762300 \\ \mathrm{Li} & 1.18216200 & 3.68014300 & -2.68473200 \\ \mathrm{O} & 4.33404200 & 1.06406200 & -3.49742800 \\ \mathrm{O} & 3.59532500 & 1.19768400 & -4.58671000 \\ \mathrm{Li} & 3.51070100 & -0.59185300 & -3.95073700 \\ \mathrm{Li} & 2.35305400 & 2.67506400 & -4.27030300 \\ \mathrm{Li} & 1.42341300 & 1.53233500 & -1.95621700 \\ \mathrm{O} & 1.89458000 & -0.89027700 & -3.25989700 \\ \mathrm{Li} & 0.19526200 & -0.98885100 & -2.30328300 \\ \mathrm{O} & 0.72972800 & 2.15464700 & -3.65217600 \\ \mathrm{Li} & -0.94783300 & 1.65746000 & -3.82536600 \\ \mathrm{Li} & 1.03053100 & 0.44101600 & -4.22812600 \\ \mathrm{O} & -0.87169500 & -0.10666600 & -4.62739600 \\ \mathrm{O} & -1.21755100 & -0.16862500 & -3.34942800 \\ \mathrm{O} & 0.84082700 & 0.07936200 & -1.00818000\end{array}$



$\mathrm{H}$
0.71252400
$0.10127700-0.05908100$
$-0.70170400-2.32533900$

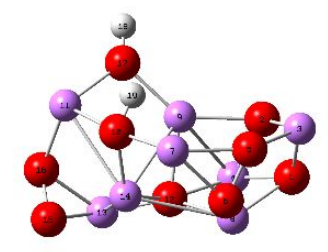

$\mathrm{Li}_{2} \mathrm{O}_{2} \_\mathrm{H}_{2} \mathrm{O} \_\mathrm{Li}(14)$ other site
03

$\begin{array}{lccc}\mathrm{O} & 2.79076400 & 2.83857300 & -3.76742700 \\ \mathrm{O} & 2.40575500 & 2.67767300 & -2.32103100 \\ \mathrm{Li} & 4.12890500 & 2.24881300 & -2.68948200 \\ \mathrm{Li} & 1.11229200 & 3.56607600 & -3.19894900 \\ \mathrm{O} & 4.43086400 & 0.34606300 & -2.27964100 \\ \mathrm{O} & 4.92919000 & 0.61846500 & -3.48613500 \\ \mathrm{Li} & 3.53074400 & -0.60863300 & -3.59584800 \\ \mathrm{Li} & 1.63389400 & 2.45727000 & -5.21057600 \\ \mathrm{Li} & 1.54209100 & 1.32735600 & -3.24951900 \\ \mathrm{O} & 1.79419600 & -0.43728400 & -4.01998700 \\ \mathrm{Li} & 0.18979700 & -1.16725300 & -3.76481300 \\ \mathrm{O} & 0.07264900 & 2.37310000 & -4.06417400 \\ \mathrm{Li} & -1.27140200 & 1.71226900 & -3.21797200 \\ \mathrm{Li} & -0.30587100 & 1.27066800 & -5.44970400 \\ \mathrm{O} & -1.34831000 & -0.19417600 & -4.36559400 \\ \mathrm{O} & -1.27427400 & -0.18738100 & -3.04156800 \\ \mathrm{O} & 1.22991300 & 1.06717500 & -6.32725100 \\ \mathrm{H} & 1.53489500 & 0.82224600 & -7.20053900 \\ \mathrm{H} & 1.73943200 & -0.08376100 & -4.93762800\end{array}$

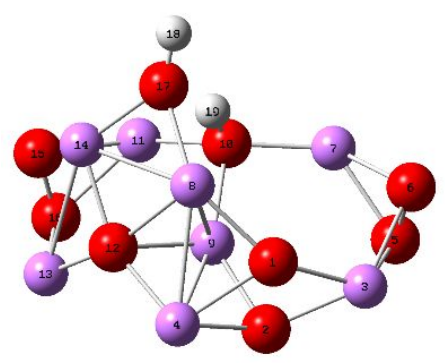

$\mathrm{Li}_{2} \mathrm{O}_{2} \_\mathrm{H}_{2} \mathrm{O} \_\mathrm{Li}(7)$ other site

03

$\begin{array}{llll}\mathrm{O} & 3.03642300 & 3.72035400 & -3.14098000 \\ \mathrm{O} & 2.37235500 & 3.15444600 & -1.90694100 \\ \mathrm{Li} & 4.14466800 & 3.06962100 & -1.90323500 \\ \mathrm{Li} & 1.25966500 & 3.34781000 & -3.36038700 \\ \mathrm{O} & 4.84353600 & 1.27825400 & -2.20654900\end{array}$




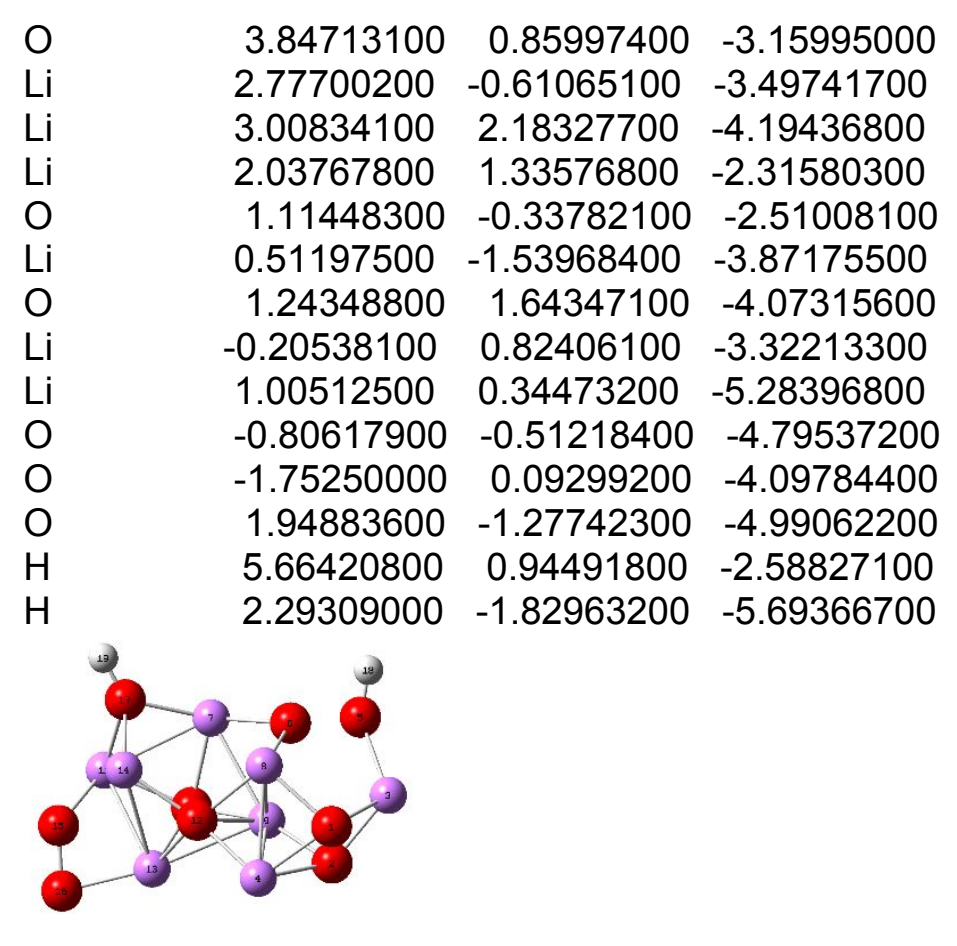

\section{S5. Energy profile corresponding to Figure 3}

(LI2O2)4-H2O SMD(DME)/M06-2X/6-31+G(d,p)

$\begin{array}{lccc}03 & & & \\ \mathrm{O} & 2.89928100 & 3.55558800 & -2.67194400 \\ \mathrm{O} & 2.38269100 & 2.54603900 & -1.68940500 \\ \mathrm{Li} & 4.14052900 & 2.37937200 & -2.10330300 \\ \mathrm{Li} & 1.01412000 & 3.58045900 & -2.84452100 \\ \mathrm{O} & 4.36120300 & 0.86189000 & -3.27084700 \\ \mathrm{O} & 3.75978100 & 1.12553200 & -4.41993800 \\ \mathrm{Li} & 3.15101900 & -0.62592500 & -3.62657300 \\ \mathrm{Li} & 2.52298900 & 2.68119900 & -4.30186500 \\ \mathrm{Li} & 1.39551700 & 1.16358600 & -2.63065700 \\ \mathrm{O} & 1.44561700 & -0.52639100 & -3.35549600 \\ \mathrm{Li} & -0.18414600 & -1.12533700 & -3.26640100 \\ \mathrm{O} & 0.75855200 & 2.27735600 & -4.10500500 \\ \mathrm{Li} & -0.97594000 & 1.95746100 & -4.19276800 \\ \mathrm{Li} & 0.94141900 & 0.56251900 & -4.71278200 \\ \mathrm{O} & -1.17737300 & 0.03541200 & -4.61606500 \\ \mathrm{O} & -1.55575800 & 0.28843900 & -3.36775000 \\ \mathrm{O} & 0.58880600 & 4.15436800 & -0.98539600 \\ \mathrm{H} & 0.88909400 & 5.02676300 & -0.70713700 \\ \mathrm{H} & 1.39467600 & 3.53178300 & -0.97458300\end{array}$




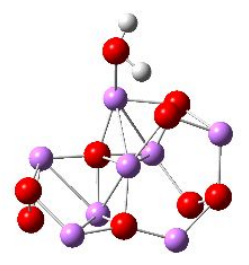

(Li2O2)4-H2O TS SMD(DME)/M06-2X/6-31+G(d,p)

03

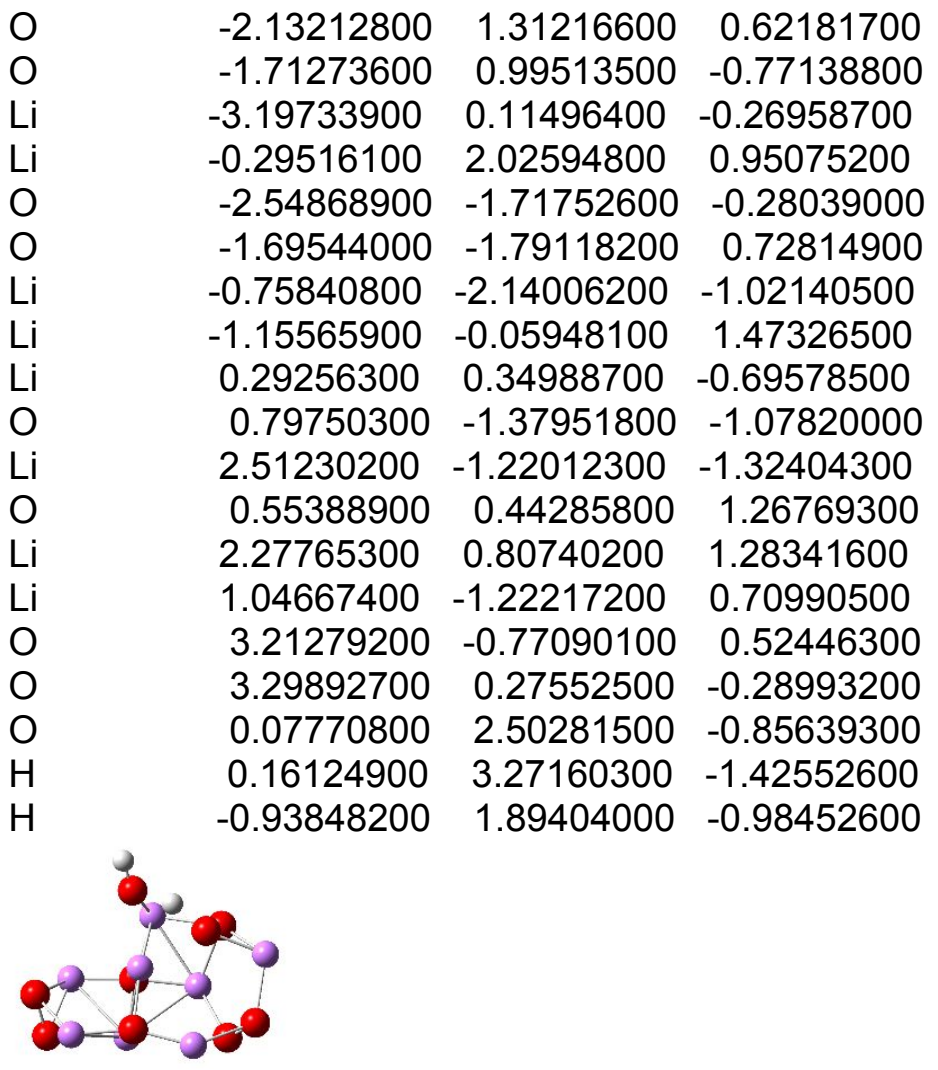

(LI2O2)4-H2O Habs product SMD(DME)/M06-2X/6-31+G(d,p)

03

$\begin{array}{llcc}\mathrm{O} & -2.32053600 & 0.73076700 & 0.78516700 \\ \mathrm{O} & -2.13143800 & 0.68561300 & -0.68224200 \\ \mathrm{Li} & -3.21422400 & -0.66988100 & 0.03843200 \\ \mathrm{Li} & -0.65733100 & 1.84799200 & 1.10051700 \\ \mathrm{O} & -1.88514900 & -2.03353700 & -0.33846000 \\ \mathrm{O} & -0.99800700 & -1.99452700 & 0.63599300 \\ \mathrm{Li} & -0.88352500 & -0.75392800 & -1.55074100 \\ \mathrm{Li} & -0.98801900 & -0.34517100 & 1.64217200 \\ \mathrm{Li} & 0.73541700 & 1.03665500 & -0.59121600 \\ \mathrm{O} & 0.84466700 & -0.53328300 & -1.51043300 \\ \mathrm{Li} & 2.57683200 & -0.64475400 & -1.55767000 \\ \mathrm{O} & 0.55197000 & 0.47643200 & 1.28848000\end{array}$




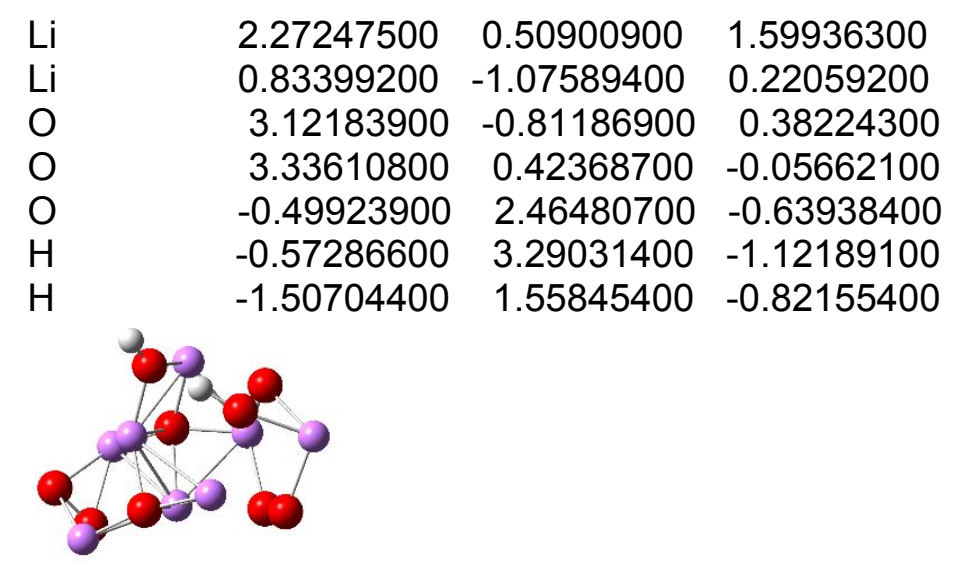

S6. Energy profile corresponding to Figure 4

Intermediate (LI2O2)4-DME SMD(DME)/M06-2X/6-31+G(d,p)

$\begin{array}{cccc}03 & & & \\ \mathrm{O} & 1.95320200 & 3.37856500 & -2.55069500 \\ \mathrm{O} & 1.23956400 & 2.18247000 & -1.97971000 \\ \mathrm{Li} & 3.00633700 & 2.28434600 & -1.55199700 \\ \mathrm{Li} & 0.37248900 & 3.00933400 & -3.40551300 \\ \mathrm{O} & 3.98304900 & 1.00610000 & -2.66512000 \\ \mathrm{O} & 3.86981700 & 1.30744200 & -3.94900800 \\ \mathrm{Li} & 3.34392300 & -0.60621500 & -3.52884200 \\ \mathrm{Li} & 2.44272600 & 2.67197300 & -4.21531800 \\ \mathrm{Li} & 1.06943700 & 0.76314400 & -3.26217600 \\ \mathrm{O} & 1.69425900 & -0.80037700 & -4.00684800 \\ \mathrm{Li} & 0.32220300 & -1.60486800 & -4.70622800 \\ \mathrm{O} & 0.87437600 & 1.95803800 & -4.82309200 \\ \mathrm{Li} & -0.53581500 & 1.41292600 & -5.71900000 \\ \mathrm{Li} & 1.65250200 & 0.39862500 & -5.36461300 \\ \mathrm{O} & -0.18530600 & -0.44869400 & -6.29054000 \\ \mathrm{O} & -1.11850500 & -0.41636700 & -5.34499500 \\ \mathrm{O} & 3.98113300 & 2.83356100 & 0.02398200 \\ \mathrm{C} & 3.51142300 & 4.01926900 & 0.65595500 \\ \mathrm{C} & 5.39838600 & 2.80201900 & -0.08231500 \\ \mathrm{H} & 3.97553700 & 4.11306300 & 1.64718200 \\ \mathrm{H} & 3.78473200 & 4.89705700 & 0.05398800 \\ \mathrm{C} & 2.00421200 & 3.91802000 & 0.78068400 \\ \mathrm{H} & 5.67237300 & 1.85867300 & -0.55724600 \\ \mathrm{H} & 5.76030300 & 3.63867500 & -0.69304900 \\ \mathrm{H} & 5.85462800 & 2.85668100 & 0.91316800 \\ \mathrm{H} & 1.73760200 & 3.03730400 & 1.38677100 \\ \mathrm{H} & 1.55202300 & 3.80628800 & -0.21599400 \\ \mathrm{O} & 1.56296400 & 5.10255200 & 1.41242700 \\ \mathrm{C} & 0.15782900 & 5.13728800 & 1.53593400 \\ \mathrm{H} & -0.10843000 & 6.08186900 & 2.01492300 \\ \mathrm{H} & -0.32869700 & 5.08089500 & 0.55205300 \\ \mathrm{H} & -0.20655100 & 4.30542200 & 2.15578900\end{array}$




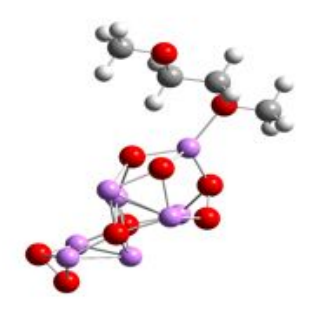

(LI2O2)4-DME TS SMD(DME)/M06-2X/6-31+G(d,p)

\begin{tabular}{lccc}
03 & & & \\
$\mathrm{O}$ & -1.32239500 & -0.16206700 & 1.89407200 \\
$\mathrm{O}$ & -0.34087800 & -0.46350400 & -1.17189600 \\
$\mathrm{Li}$ & -0.89547700 & -1.33861000 & 0.44905900 \\
$\mathrm{Li}$ & -0.50053200 & 1.32702500 & -0.71769800 \\
$\mathrm{O}$ & 0.97064900 & -1.50424500 & 1.30573500 \\
$\mathrm{O}$ & 2.03108600 & -1.59178900 & 0.33380400 \\
$\mathrm{Li}$ & 2.41412200 & -1.85069600 & 2.38480600 \\
$\mathrm{Li}$ & 0.37693400 & 0.37963200 & 1.40456400 \\
$\mathrm{Li}$ & 1.50883000 & -0.23147800 & -1.01990100 \\
$\mathrm{O}$ & 3.20116300 & -0.98801400 & 0.95261500 \\
$\mathrm{Li}$ & 4.23913000 & -0.50118800 & -0.59940500 \\
$\mathrm{O}$ & 1.12229000 & 1.34587500 & 0.07332500 \\
$\mathrm{Li}$ & 2.29007700 & 1.98741500 & -1.19385800 \\
$\mathrm{Li}$ & 2.78184000 & 0.90490200 & 0.70463500 \\
$\mathrm{O}$ & 3.93191900 & 1.28648900 & -0.75293200 \\
$\mathrm{O}$ & 3.17696400 & 0.42411400 & -1.73010400 \\
$\mathrm{O}$ & -2.83102400 & -1.53724000 & -0.26924600 \\
$\mathrm{C}$ & -3.30733500 & -0.26481000 & -0.70051700 \\
$\mathrm{C}$ & -2.90556900 & -2.49234100 & -1.31351500 \\
$\mathrm{C}$ & -3.04225000 & 0.78495000 & 0.36636500 \\
$\mathrm{H}$ & -2.78771600 & 0.02379900 & -1.62396700 \\
$\mathrm{H}$ & -4.38211800 & -0.34932800 & -0.91314500 \\
$\mathrm{H}$ & -2.57297400 & -3.45048700 & -0.90874000 \\
$\mathrm{H}$ & -3.93609400 & -2.59613700 & -1.67760400 \\
$\mathrm{H}$ & -2.25438100 & -2.20154000 & -2.14966800 \\
$\mathrm{H}$ & -2.36044900 & 0.29842500 & 1.23377500 \\
$\mathrm{H}$ & -3.95620500 & 1.15516400 & 0.84764500 \\
$\mathrm{O}$ & -2.32374400 & 1.87420700 & -0.18861800 \\
$\mathrm{C}$ & -2.20961300 & 2.95661400 & 0.73204100 \\
$\mathrm{H}$ & -1.64312500 & 3.74830100 & 0.23824500 \\
$\mathrm{H}$ & -3.20478800 & 3.33299000 & 0.99738300 \\
$\mathrm{H}$ & -1.68656900 & 2.63071000 & 1.63917500 \\
$\mathrm{H}$ & & & \\
\hline & & &
\end{tabular}


(LI2O2)H-DME product SMD(DME)/M06-2X/6-31+G(d,p)

\begin{tabular}{cccc}
03 & & & \\
$\mathrm{O}$ & 1.07503700 & -0.30681100 & 1.76101000 \\
$\mathrm{O}$ & 0.33879900 & 0.11234100 & -1.09337300 \\
$\mathrm{Li}$ & 0.75234600 & 1.06749400 & 0.50877200 \\
$\mathrm{Li}$ & 0.64603000 & -1.31516900 & 0.14572800 \\
$\mathrm{O}$ & -1.10915600 & 1.49401000 & 1.28811600 \\
$\mathrm{O}$ & -2.07635000 & 1.50243400 & 0.21590000 \\
$\mathrm{Li}$ & -2.66656400 & 2.16185600 & 2.00827200 \\
$\mathrm{Li}$ & -0.71191900 & -0.38552800 & 1.75365500 \\
$\mathrm{Li}$ & -1.47284000 & -0.04142600 & -0.91258300 \\
$\mathrm{O}$ & -3.31060700 & 0.95509300 & 0.76390400 \\
$\mathrm{Li}$ & -4.19635200 & 0.30504500 & -0.84337900 \\
$\mathrm{O}$ & -1.22746100 & -1.44583700 & 0.38714400 \\
$\mathrm{Li}$ & -2.20878200 & -2.21903300 & -0.95312100 \\
$\mathrm{Li}$ & -2.95425700 & -0.97123800 & 0.74899700 \\
$\mathrm{O}$ & -3.91553600 & -1.49309900 & -0.77631900 \\
$\mathrm{O}$ & -3.04806500 & -0.74108400 & -1.74903800 \\
$\mathrm{O}$ & 2.52283900 & 1.70514600 & -0.18906300 \\
$\mathrm{C}$ & 3.21972200 & 0.67579700 & -0.92548400 \\
$\mathrm{C}$ & 2.28710100 & 2.83694200 & -1.01349100 \\
$\mathrm{C}$ & 3.51970200 & -0.50674600 & -0.09538400 \\
$\mathrm{H}$ & 2.61058900 & 0.37548000 & -1.78692800 \\
$\mathrm{H}$ & 4.15950100 & 1.12350500 & -1.28267200 \\
$\mathrm{H}$ & 1.77969500 & 3.59006100 & -0.40617600 \\
$\mathrm{H}$ & 3.23285800 & 3.25525900 & -1.38213300 \\
$\mathrm{H}$ & 1.65287900 & 2.57154700 & -1.87018100 \\
$\mathrm{H}$ & 1.87283000 & -0.28555600 & 2.28940500 \\
$\mathrm{H}$ & 4.15648000 & -0.45931600 & 0.78325900 \\
$\mathrm{O}$ & 2.75987400 & -1.62321700 & -0.25908300 \\
$\mathrm{C}$ & 3.16218700 & -2.71504400 & 0.56591100 \\
$\mathrm{H}$ & 2.51951400 & -3.56055200 & 0.31634300 \\
$\mathrm{H}$ & 4.20596200 & -2.97710000 & 0.36339700 \\
$\mathrm{H}$ & 3.03943500 & -2.45683200 & 1.62376000 \\
& & & \\
\hline & & & \\
& & &
\end{tabular}


S7. Mulliken charge analysis for $\left.\mathrm{Li}_{8}(\mathrm{OH})_{8}-1\right]^{-}$

\begin{tabular}{|c|c|c|}
\hline $\begin{array}{c}\text { Atom } \\
\text { number }\end{array}$ & Element & $\begin{array}{c}\text { Mulliken } \\
\text { charge }\end{array}$ \\
\hline 1 & 0 & -0.707 \\
\hline 2 & 0 & -0.772 \\
\hline 3 & $\mathrm{Li}$ & 0.787 \\
\hline 4 & $\mathrm{Li}$ & 1.572 \\
\hline 5 & $\mathrm{Li}$ & -0.770 \\
\hline 6 & $\mathrm{Li}$ & 1.308 \\
\hline 7 & $\mathrm{Li}$ & 1.735 \\
\hline 8 & 0 & -0.643 \\
\hline 9 & $\mathrm{Li}$ & -2.129 \\
\hline 10 & 0 & -0.692 \\
\hline 11 & $\mathrm{Li}$ & -1.688 \\
\hline 12 & $\mathrm{Li}$ & 0.885 \\
\hline 13 & 0 & -0.724 \\
\hline 14 & $\mathrm{H}$ & 0.393 \\
\hline 15 & $\mathrm{H}$ & 0.389 \\
\hline 16 & 0 & -0.649 \\
\hline 17 & $\mathrm{H}$ & 0.430 \\
\hline 18 & $\mathrm{H}$ & 0.421 \\
\hline 19 & 0 & -0.690 \\
\hline 20 & $\mathrm{H}$ & 0.329 \\
\hline 21 & $\mathrm{H}$ & 0.424 \\
\hline 22 & 0 & -0.674 \\
\hline 23 & $\mathrm{H}$ & 0.371 \\
\hline 24 & $\mathrm{H}$ & 0.420 \\
\hline 25 & 1 & -0.323 \\
\hline
\end{tabular}

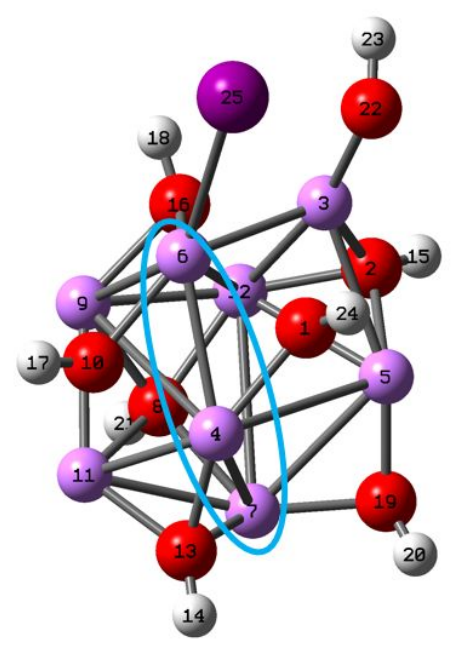

